This item was submitted to Loughborough's Research Repository by the author.

Items in Figshare are protected by copyright, with all rights reserved, unless otherwise indicated.

\title{
Situation awareness for UAV operating in terminal areas using bearing-only observations and circuit flight rules
}

PLEASE CITE THE PUBLISHED VERSION

http://dx.doi.org/10.1109/ACC.2016.7524960

PUBLISHER

IEEE / @ American Automatic Control Council (AACC)

VERSION

AM (Accepted Manuscript)

LICENCE

CC BY-NC-ND 4.0

\section{REPOSITORY RECORD}

Coombes, Matthew, Cunjia Liu, and Wen-Hua Chen. 2016. "Situation Awareness for UAV Operating in Terminal Areas Using Bearing-only Observations and Circuit Flight Rules”. figshare.

https://hdl.handle.net/2134/22097. 


\title{
Situation awareness for UAV operating in terminal areas using bearing-only observations and circuit flight rules
}

\author{
Matthew Coombes, Cunjia Liu, Wen-Hua Chen \\ Department of Automotive and Aeronautical Engineering \\ Loughborough University, Loughborough, LE11 3TQ UK \\ Email: \{M.J.Coombes@lboro.ac.uk, C.Liu@lboro.ac.uk, W.Chen@lboro.ac.uk\}
}

\begin{abstract}
Situation awareness is required for an Unmanned Aerial Vehicle (UAV) when it makes an arrival at an uncontrolled airfield. Since no air traffic control service is available, the UAV needs to detect and track other traffic aircraft by using its onboard sensors. General aviation pilots obtain enough situation awareness to operate in these environments, only using their vision and radio messages heard from other traffic aircraft. To improve the target tracking performance of a UAV, the circuit flight rules and standard radio messages are incorporated to provide extra knowledge about the target behaviour. This is achieved by using the multiple models to describe the target motions in different flight phases and characterising the phase transition in a stochastic manner. Consequently, an interacting multiple model particle filter with state-dependent transition probabilities is developed to perform Bayesian filtering with bearing-only observations from a vision sensor.
\end{abstract}

\section{INTRODUCTION}

There is a large drive for Unmanned Aerial Vehicles (UAVs) to be integrated within the national airspace system. This is due to the huge advantages of their use in areas such as search and rescue, border security and environmental monitoring. However, Civil Aviation Authority in U.K. has stated in CAP722 that "For a UAV to operate within busy civil airspace it is required to comply with Air Traffic Control (ATC) instruction in the same way and within the same timeframe that the pilot of a manned aircraft would" [1]. This means that a UAV must be predictable, communicative, perform self-separation and see-and-avoid against other air traffic. These requirements become more critical when a UAV is flying into a terminal area around an airport with high traffic density, which in turn necessitates the development of advanced situation awareness for the UAV.

The scenario concerned in this paper is that a UAV is making an arrival into an uncontrolled licensed aerodrome. Unlike a controlled aerodrome where ATC is responsible for all separation between aircraft and their navigation, in an uncontrolled aerodrome the service is completely non authoritative. Therefore, the pilots, or equivalently UAVs, are completely responsible for their own operations. Smaller aircraft operating in and around uncontrolled aerodromes are unlikely to have Automated Dependant Surveillance Broadcast (ADS-B) system, which can directly transmit their state to all other aircraft. This means the UAV has to rely solely on its onboard sensors, like a monocular camera, to detect and track traffic aircraft thus to build good situation awareness. Specifically, the UAV needs to be able to track other traffic aircraft, know there intentions and stage of flight, and project their positions to the future in order to maintain adequate separation.

It is known that estimating other aircraft's state using the onboard sensors can be cast into a target tracking problem and solved by different filtering techniques [2]. Given camera based vision systems are commonly equipped by UAVs, a bearing only tracking algorithm is investigated in this paper comparing to a previous study [3]. Bearings-only tracking presents a challenge for any manouvering target tracking algorithm due to target's low observability and inherent nonlinearities [4]. To improve tracking performance in bearing only tracking, [5] suggests that the observer vehicle must significantly out manoeuvre the target. However, in the terminal area the UAV will not be allowed to manoeuvre how it likes, as it will need to fly safely and predictably in accordance with the rules of the air, just as the traffic aircraft will be.

To improve the situation awareness of a UAV in uncontrolled aerodrome, this paper aims to incorporate aerodrome circuit flight rules, and some of the standard radio messages transmitted from other traffic aircraft into the filtering process. Therefore, the knowledge of the circuit pattern and these radio messages need to be converted into a probabilistic representation to be exploited by the filtering process. First, multiple models of aircraft dynamics are adopted to characterise the behaviours at different flight phases. To further reflect the flight direction in each circuit phase, minimum velocity constraints are applied on each model by taking into account the stall speed of the aircraft, as well as lateral velocity constrains to reflect that the circuit legs are approximately parallel or perpendicular to the runway. Second, the transition behaviours between consecutive flight phases are described in a state-dependent and stochastic manner. Compared with the traditional Markov jump transition techniques (see, e.g. ref [6]), the state-dependent transition (SDT) allows one to more precisely describe a phase transition as stated in flight rules, whereas the stochastic modelling is able to capture the uncertainties during this process. Thirdly the use of the small number of radio messages as an extra and different measurement, to further enhance the information provided to the filter.

It is a challenging task to solve the corresponding multimode target tracking problem after incorporating the circuit knowledge due to the nonlinear/non-Gaussian nature of this 
kind of problems. Interacting multiple model (IMM) schemes based on the Kalman filters [6], [7] and particle filter (PF) solutions [8], [9] have been developed for Markov jump transition. When the transition behaviour are modelled as a linear state-dependent function with Gaussian stochastic uncertainties, Seah and Hwang have developed a promising hybrid estimation framework based on multiple Kalman filters under Gaussian assumptions [10], [11]. However, when both the system models and the state-dependent transition are in nonlinear forms, Kalman filter based algorithms are no longer applicable. To this end, this paper follows a general IMM Bayesian filtering framework [12] to develop the filtering cycle. To actually implement the Bayesian framework for the formulated target tracking problem, a constrained particle filter solution is developed, which is able to take into account the state constraints and nonlinear stochastic transition of system modes.

\section{VISUAL FLIGHT RULES IN TERMINAL AREAS}

A terminal area is the airspace surrounding an airfield which is mainly used by aircraft arriving at or departing from that airfield, which can be either controlled or uncontrolled. Uncontrolled airfields will normally be smaller and have non-authoritative Aircraft Flight Information Service (AFIS) instead of an ATC. At these aerodromes the pilot, or equivalently a UAV, is completely responsible for their own operations and no clearances are required, hence a high level of situation awareness is needed. Given no ATC service available, aircraft landing at an uncontrolled aerodrome are required to follow the Visual Flight Rule (VFR), which consists of a number of standard procedures. The circuit flight pattern is the most significant VFR procedure. The circuit is a rectangular flight path flown by all arriving aircraft which ensures that the runway and all other traffic remain in sight at all times. There are four distinct phases of the circuit, namely crosswind leg, downwind leg, base leg and final, which are flown in that order [13]. These are shown in Fig. 1.

The use of VHF radio messages is also an essential part of aerodrome operations, used by both the ground radio operators, and other aircraft to give locations as well as intentions. These messages are very standard in nature for example

$$
\begin{aligned}
& \text { G-ABCD, Downwind to land } \\
& \text { G-ABCD, report final } \\
& \text { WILCO, G-ABCD }
\end{aligned}
$$

This is the radio message transmitted by the aircraft with registration $\mathrm{G}-\mathrm{ABCD}$ saying that they have reached the downwind part of the circuit. This tells any other pilot the aircraft's approximate location relative to the runway and that the aircraft will flying parallel to the runway opposite to the landing direction.

The exact dimensions of a circuit are not completely fixed but can be greatly constrained by a couple of rules.
For example, the downwind leg is flown parallel to the runway, until the aircraft is at a relative bearing of $45^{\circ}$ from the threshold point of the runway from the extended centreline [14], where another $90^{\circ}$ turn is made to the base leg. Somewhere around the beginning of the base leg the aircraft will start descending, and slowing until the aircraft reaches the speed where it can extend its flaps. Next, a turn onto the final leg to land will be performed. By the end of the turn the aircraft is flying straight at the runway down the extended centreline. When the aircraft is lined up on final it will make another radio call. Fig. 1 shows the approximate areas where both the downwind and final radio calls will be made. It can be seen that the area is more well defined for the call on final than downwind as an aircraft will be fly the final leg close to the extended centreline.

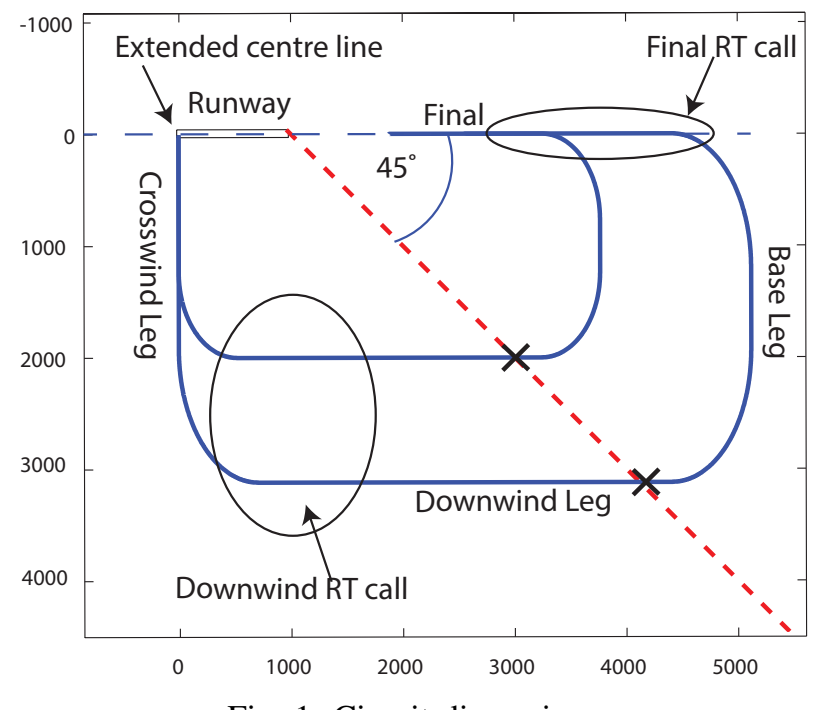

Fig. 1: Circuit dimensions

For a UAV intending to land at an uncontrolled airfield, it needs to be aware of other aircraft within this terminal region, especially those who are already in the circuit and may breach the separation criterion in the near future. In order to coordinate with other aircraft to guarantee the selfseparation, the essential problem of situation awareness in terminal region is to acquire the status of target aircraft and their intentions, i.e. the circuit phases. Generally, this problem can be cast into a target tracking problem based on the understanding of the target's motion and the observation from sensors.

\section{PROBLEM FORMULATION}

The target tracking using bearing only measurements, Radiotelephony (RT) and circuit flight rules is formulated in this section. First, the aircraft's dynamics associated with each fight phase are modelled where the phase transition behaviour is described in a state-dependent stochastic form. Then, in conjunction with the sensor model, the estimation problem is formulated in a filtering problem. 


\section{A. Aircraft model}

To estimate the state of a traffic aircraft in the circuit, a mathematical model is required to describe its dynamical behaviour. Although in general the aircraft's state can be described by position and velocity, the aircraft may possess different dynamic features in different circuit phases. Therefore, multiple models are adopted according to different circuit phases. Because circuit height is usually pre-determined and as collisions are more likely to occur when aircraft fly at the same height, this paper only focuses on the horizontal motions of the aircraft in 2-dimensions. Given the aircraft position $(x, y)$ in local coordinates, the aircraft state at time index $k$ can be defined as $\mathbf{x}_{k}=\left[\begin{array}{llll}x_{k} & \dot{x}_{k} & y_{k} & \dot{y}_{k}\end{array}\right]^{T}$, which also includes the velocity. A number of candidate models are summarised as follows.

1) Constant velocity model: The aircraft flying in the crosswind and downwind leg can be described by the constant velocity $(\mathrm{CV})$ model, such that

$$
\mathbf{x}_{k+1}=\left[\begin{array}{cccc}
1 & T & 0 & 0 \\
0 & 1 & 0 & 0 \\
0 & 0 & 1 & T \\
0 & 0 & 0 & 1
\end{array}\right] \mathbf{x}_{k}+\left[\begin{array}{cc}
\frac{T^{2}}{2} & 0 \\
T & 0 \\
0 & \frac{T^{2}}{2} \\
0 & T
\end{array}\right] \mathbf{w}_{k}
$$

where $k$ is the time index, $T$ is the sampling time, $\mathbf{x}_{k} \in \mathbb{R}^{4}$ is the aircraft state, and $\mathbf{w}_{k} \in \mathbb{R}^{2}$ is the process noise. The noise vector $\mathbf{w}_{k}$ is assumed to be Gaussian with zero mean vector and covariance matrix $Q_{k}$.

2) White-noise acceleration model: The aircraft flying in base leg and final approach are more likely to reduce their speed rather than maintaining a constant cruise. The simplest model for describing this motion is a white-noise acceleration (WA) model, which differs from the CV model (1) only in the noise level [15].

3) Coordinated turn model: The model associated with the turning phases, including turning onto the downwind leg, base leg and final approach, can be described by the coordinated turn (CT) model

$$
\begin{aligned}
\mathbf{x}_{k+1}= & {\left[\begin{array}{cccc}
1 & \frac{\sin (\omega T)}{\omega} & 0 & -\frac{1-\cos (\omega T)}{\omega} \\
0 & \cos (\omega T) & 0 & -\sin (\omega T) \\
0 & \frac{1-\cos (\omega T)}{\omega} & 1 & \frac{\sin (\omega T)}{\omega} \\
0 & \sin (\omega T) & 0 & \cos (\omega T)
\end{array}\right] \mathbf{x}_{k} } \\
& +\left[\begin{array}{cc}
\frac{T^{2}}{2} & 0 \\
T & 0 \\
0 & \frac{T^{2}}{2} \\
0 & T
\end{array}\right] \mathbf{w}_{k}
\end{aligned}
$$

where $\omega$ is the nominal turning rate and the Gaussian process noise $\mathbf{w}_{k}$ is used to capture the uncertainties.

In addition to the multiple models which are used to distinguish between the flight phases, other information on circuit flight like the speed constraints can also be exploited to improve the target tracking.

\section{B. Discrete mode transition}

To incorporate the multiple models associated with the circuit flight, a discrete mode variable $m_{k} \in \mathcal{M}$ is introduced to indicate the current phase of the aircraft, where
$\mathcal{M}=\{1,2, \ldots, M\}$ is the set of $M$ circuit phases. From the crosswind to final approach, the circuit phases and the corresponding models are summarised in Table I.

TABLE I: Circuit phases and aircraft models.

\begin{tabular}{c|l|c}
\hline Mode & Circuit phase & Aircraft Model \\
\hline 1 & crosswind & $\mathrm{CV}$ \\
2 & turning into downwind & $\mathrm{CT}$ \\
3 & downwind & $\mathrm{CV}$ \\
4 & turning into base & $\mathrm{CT}$ \\
5 & base & WA \\
6 & turning into final & $\mathrm{CT}$ \\
7 & final & WA \\
\hline
\end{tabular}

The mode transition process can be described in a probabilistic way. Suppose at time $k$ the transition probability from mode $i$ to mode $j$ is denoted as $\pi_{i j}\left(\mathbf{x}_{k}\right)$, where $i, j \in \mathcal{M}$. A commonly used method for modelling this process assumes the Markov jump model where the probability $\pi_{i j}$ is assumed to be a known constant and independent of the state $\mathbf{x}_{k}$. Although this method is very popular in the target tracking field, it cannot fully take into account the flight patterns in the circuit. As mentioned earlier there is no nominal circuit path for all aircraft, the pilots usually execute the circuit flight by following a number of rules. Therefore, it is necessary to develop a dedicated transition model for the circuit flight.

In general, the stochastic transition criterion from mode $i$ to mode $j$ can be modelled as a nonlinear function of state $\mathbf{x}_{k}$ in conjunction with a random vector to capture the uncertainties (see [3]), such that

$$
\mathbf{G}_{i j}\left(\mathbf{x}_{k}\right)+\gamma_{i j} \leq 0,
$$

where $G_{i j}\left(\mathbf{x}_{k}\right)$ is a nonlinear function of $\mathbf{x}_{k}$ and $\gamma_{i j}$ is a random vector satisfying a distribution $p_{i j}(\gamma)$. The mode transition probability thus can be expressed as

$$
\pi_{i j}\left(\mathbf{x}_{k}\right)=\operatorname{Pr}\left\{G_{i j}\left(\mathbf{x}_{k}\right)+\gamma_{i j} \leq 0\right\}
$$

In particular, when $\gamma_{i j}$ follows a Gaussian distribution with zero mean and standard deviation of $\sigma$, (4) can be written as

$$
\pi_{i j}\left(\mathbf{x}_{k}\right)=\Phi\left(-G_{i j}\left(\mathbf{x}_{k}\right) ; 0, \sigma^{2}\right)=1-\Phi\left(G_{i j}\left(\mathbf{x}_{k}\right) ; 0, \sigma^{2}\right),
$$

where $\Phi\left(y ; 0, \sigma^{2}\right)=\int_{\infty}^{y} \mathcal{N}\left(u ; 0, \sigma^{2}\right) \mathrm{d} u$ denotes the cumulative distribution function (CDF) with zero mean and standard deviation $\sigma$. The constructed mode transition probabilities need to be normalised such that $\sum_{j \in \mathcal{M}} \pi_{i j}\left(\mathbf{x}_{k}\right)=1$. Other distributions for random variable $\gamma i j$ may also be adopted depending on the scenario, but it is beyond the scope of the current paper.

The state-dependent transition has been investigated in some pioneering works, including an example where the transition probability $\pi_{i j}\left(\mathbf{x}_{k}\right)$ is a piecewise linear function of state $\mathbf{x}_{k}$ [12] and multiple Markov jump probabilities depending on the system state [16]. A more advanced modelling of using random variables to capture the transition uncertainties has been shown in a series of hybrid state estimation algorithms [10], [11], where the state-dependent function and the integration of the random variables have 
to satisfy a linear form. Comparing to above methods, this paper adopts a general nonlinear state function to provide more flexibilities.

\section{Hybrid state estimation}

Combining the multiple models described in Eq. (1)-(2) and the transition of the discrete mode variable $m_{k}$, the motions and intentions of the traffic aircraft in the circuit can be cast into a hybrid system

$$
\mathbf{x}_{k+1}=\mathbf{f}\left(\mathbf{x}_{k}, m_{k}, \mathbf{w}_{k}\right), \quad m_{k} \in \mathcal{M}
$$

where the discrete state $m_{k}$ determines the aircraft model and the associated process noise $\mathbf{w}_{k}$ that govern the evolution of continuous state $\mathbf{x}_{k}$.

In order to estimate the traffic aircraft's state described in Eq. (6) without any direct measurements from transponders, a UAV needs to rely on bearing information from the camera, and may use RT information to infer the position and intentions of traffic aircraft. Therefore, in this paper the measurement model is expressed as.

$$
\mathbf{z}_{k}=\left[\begin{array}{c}
x_{k} \\
y_{k} \\
\arctan \left(\frac{y_{k}-\bar{y}_{k}}{x_{k}-\bar{x}_{k}}\right)
\end{array}\right]+\mathbf{v}_{k}
$$

where $\left(\bar{x}_{k}, \bar{y}_{k}\right)$ denotes the UAV's position assumed to be available, and $\mathbf{v}_{k}$ denotes the observation noise vector which is Gaussian with zero mean vector and covariance matrix $R$. The first two elements in $\mathbf{v}_{k}$ represent the uncertainty in the RT location report, and the final element represents the actual noise on the bearing measurement to the traffic aircraft. However as radio calls are not very frequent, between them only the bearing part of the measurement will be used.

The current situation of the traffic aircraft can be represented by the probabilistic distribution of the aircraft's state and the associated mode. Therefore, the awareness of the aircraft involves the estimate of the distribution $p\left(\mathbf{x}_{k}, m_{k} \mid \mathbf{z}_{1: k}\right)$ at time $k$ based on the observation sequence $\mathbf{z}_{1: k}=\left\{\mathbf{z}_{1}, \ldots, \mathbf{z}_{k}\right\}$ and the initial distribution $p\left(\mathbf{x}_{0}, m_{0}\right)$, which in turn can be done in a recursive probabilistic inference process.

\section{BAYESIAN FILTERING}

Bayesian filtering provides a generic mathematical tool for probabilistic inference. However, for the formulated hybrid system a dedicated IMM Bayesian filtering scheme in [12] will be adopted with a particle filter implementation.

\section{A. IMM Bayesian framework}

Baye's theorem can be used to construct the conditional density $p\left(\mathbf{x}_{k}, m_{k} \mid \mathbf{z}_{1: k}\right)$. Following [12], the Bayesian inference can be summarised as

$$
\begin{array}{r}
p\left(\mathbf{x}_{k}, m_{k}^{j} \mid \mathbf{z}_{1: k}\right)=\frac{1}{c_{t}} p\left(\mathbf{z}_{k} \mid \mathbf{x}_{k}, m_{k}^{j}\right) \int_{\mathbb{R}^{n}} p\left(\mathbf{x}_{k} \mid \mathbf{x}_{k-1}, m_{k}^{j}\right) \\
\sum_{i \in \mathbb{M}} \Pi_{i j}\left(\mathbf{x}_{k-1}\right) p\left(\mathbf{x}_{k-1}, m_{k-1}^{i} \mid \mathbf{z}_{1: k-1}\right) \mathrm{d} \mathbf{x}_{k-1}
\end{array}
$$

where $c_{t}$ is the normalisation factor, $m_{k}^{j}$ denotes that the discrete mode $m_{k}$ takes the value of $j, m_{k-1}^{i}$ denotes $m_{k-1}=i$ and the state-dependent transition probability is defined as

$$
\Pi_{i j}\left(\mathbf{x}_{k-1}\right)=p\left(m_{k}^{j} \mid m_{k-1}^{i}, \mathbf{x}_{k-1}\right), \quad \forall i, j \in \mathcal{M}
$$

To implement the above recursive equation by using a bank of mode-matched filters, the inference process is decomposed into four steps.

1) Mode transition: The evolution of the conditional model probability from $k-1$ to $k$ is characterised as the mode transition step, such that

$$
\begin{aligned}
& p\left(m_{k}^{j} \mid \mathbf{z}_{1: k-1}\right)=p\left(m_{k-1}^{i} \mid \mathbf{z}_{1: k-1}\right) \\
& \sum_{i \in \mathbb{M}} \int_{\mathbb{R}^{n}} \Pi_{i j}\left(\mathbf{x}_{k-1}\right) p\left(\mathbf{x}_{k-1} \mid m_{k-1}^{i}, \mathbf{z}_{1: k-1}\right) \mathrm{d} \mathbf{x}_{k-1}
\end{aligned}
$$

This relation shows that the predicted model probability $p\left(m_{k}^{j} \mid \mathbf{z}_{1: k-1}\right)$ can be derived from the mode conditional density $p\left(\mathbf{x}_{k-1} \mid m_{k-1}^{i}, \mathbf{z}_{1: k-1}\right)$ and the mode probability $p\left(m_{k-1}^{i} \mid \mathbf{z}_{1: k-1}\right)$.

2) State interaction: The initial state density $p\left(\mathbf{x}_{k-1} \mid m_{k}^{j}, \mathbf{z}_{1: k}\right)$ for each mode-matched filter is calculated in this step by considering the influence of the predicted model probability as

$p\left(\mathbf{x}_{k-1} \mid m_{k}^{j}, \mathbf{z}_{1: k}\right)=\frac{\sum_{i \in \mathbb{M}} \Pi_{i j}\left(\mathbf{x}_{k-1}\right) p\left(\mathbf{x}_{k-1}, m_{k-1}^{i} \mid \mathbf{z}_{1: k-1}\right)}{p\left(m_{k}^{j} \mid \mathbf{z}_{1: k-1}\right)}$

3) State evolution: Given the initial state for each modematched filter in Eq. (11), the state evolution step propagates these densities from $k-1$ to $k$ through integration, such that

$$
\begin{aligned}
& p\left(\mathbf{x}_{k} \mid m_{k}^{j}, \mathbf{z}_{1: k-1}\right) \\
& =\int_{\mathbb{R}^{n}} p\left(\mathbf{x}_{k} \mid \mathbf{x}_{k-1}, m_{k}^{j}\right) p\left(\mathbf{x}_{k-1} \mid m_{k}^{j}, \mathbf{z}_{1: k-1}\right) \mathrm{d} \mathbf{x}_{k-1}
\end{aligned}
$$

where the transition density $p\left(\mathbf{x}_{k} \mid \mathbf{x}_{k-1}, m_{k}^{j}\right)$ can be obtained from the corresponding models and distributions of process noises.

4) Correction: In this step, the latest measurement information is used to update the prior distribution in each mode-matched filter. Following Eq. (??) gives

$$
\begin{aligned}
& p\left(\mathbf{x}_{k}, m_{k}^{j} \mid \mathbf{z}_{1: k}\right) \\
& \propto p\left(\mathbf{z}_{k} \mid \mathbf{x}_{k}, m_{k}^{j}\right) p\left(\mathbf{x}_{k} \mid m_{k}^{j}, \mathbf{z}_{1: k-1}\right) p\left(m_{k}^{j} \mid \mathbf{z}_{1: k-1}\right)
\end{aligned}
$$

where the likelihood density $p\left(\mathbf{z}_{k} \mid \mathbf{x}_{k}, m_{k}^{j}\right)$ can be derived from the observation model.

To complete the recursive cycle, the required modeconditioned state distribution and mode probability at the next time step can also be calculated such that

$$
p\left(m_{k}^{j} \mid \mathbf{z}_{1: k}\right)=\int_{\mathbb{R}^{n}} p\left(\mathbf{x}_{k}, m_{k}^{j} \mid \mathbf{z}_{1: k}\right) \mathrm{d} \mathbf{x}_{k}
$$

and

$$
p\left(\mathbf{x}_{k} \mid m_{k}^{j}, \mathbf{z}_{1: k}\right) \propto p\left(\mathbf{z}_{k} \mid \mathbf{x}_{k}, m_{k}^{j}\right) P\left(\mathbf{x}_{k} \mid m_{k}^{j}, \mathbf{z}_{1: k-1}\right) .
$$




\section{B. Particle filter implementation}

Although the Bayesian filtering scheme provides a general framework for state estimation of the hybrid systems, a particle filter implementation is required to solve this nonlinear and non-Gaussian filtering problem.

Suppose that at time $k-1$ there exists a set of weighted particles $\left\{\mathbf{x}_{k-1}^{i, n}, \mu_{k-1}^{i, n} ; i \in \mathcal{M}, n \in\{1, \ldots, N\}\right\}$, which spans over the joint distribution

$$
p\left(\mathbf{x}_{k-1}, m_{k-1}^{i} \mid Z^{k-1}\right) \approx \sum_{n=1}^{N} \mu_{k-1}^{i, k} \delta\left(\mathbf{x}_{k-1}-\mathbf{x}_{k-1}^{i, k}\right)
$$

where $\delta(\cdot)$ is a Dirac delta function. For each discrete mode $i \in \mathcal{M}, N$ particles are allocated to the corresponding modematched filter with a total number of $N_{p}=N \cdot M$ weighted particles.

The development of Sequential Monte Carlo implementation involves the substitution of the empirical density Eq. (16) into four filtering cycles, respectively. Firstly, the prior mode probability in Eq. (10) is approximated as

$$
p\left(m_{k}^{j} \mid \mathbf{z}_{1: k-1}\right) \approx \sum_{i=1}^{M} \sum_{n=1}^{N} \pi_{i j}\left(\mathbf{x}_{k-1}^{i, n}\right) \cdot \mu_{k-1}^{i, n} \triangleq \Lambda_{k-1}^{j} .
$$

Secondly, the initial density for each mode-conditioned filter in Eq. (11) can be approximated

$$
\begin{aligned}
& p\left(\mathbf{x}_{k-1} \mid m_{k}^{j}, \mathbf{z}_{1: k-1}\right) \\
& \approx \sum_{i=1}^{M} \sum_{n=1}^{N} \pi_{i j}\left(\mathbf{x}_{k-1}^{i, n}\right) \mu_{k-1}^{i, n} \delta\left(\mathbf{x}_{k-1}-\mathbf{x}_{k-1}^{i, n}\right) / \Lambda_{k-1}^{j}
\end{aligned}
$$

where the density $p\left(\mathbf{x}_{k-1} \mid m_{k}^{j}, \mathbf{z}_{1: k-1}\right)$ is approximated by $N_{p}$ particles instead of $N$. A solution to this problem as suggested in ref [12] is to perform a resampling process so that $N$ samples $\left\{\overline{\mathbf{x}}_{k-1}^{j, n}, \bar{\mu}_{k-1}^{j, n}\right\} \sim p\left(\mathbf{x}_{k-1} \mid m_{k}^{j}, \mathbf{z}_{1: k-1}\right)$ are generated for each mode.

The third step is to propagate the mode-conditioned density derived in (18) to the next time step $k$. This means to draw new samples $\left\{\mathbf{x}_{k}^{j, n}\right\}$ from $p\left(\mathbf{x}_{k} \mid \mathbf{x}_{k-1}, m_{k}^{j}\right)$ based on the resampled particles $\left\{\overline{\mathbf{x}}_{k-1}^{j, n}, \bar{\mu}_{k-1}^{j, n}\right\}$ so that

$$
p\left(\mathbf{x}_{k} \mid \mathbf{x}_{k-1}, m_{k}^{j}\right) \approx \sum_{n=1}^{N} \bar{\mu}_{k}^{j, n} \delta\left(\mathbf{x}_{k}-\mathbf{x}_{k}^{j, n}\right) .
$$

The last step in the filtering cycle aims to update the weights using the likelihood function. Inserting Eq. (19) and the predicted mode probability $\Lambda_{k-1}^{j}$ into Eq. (13) gives

$$
\begin{aligned}
& p\left(\mathbf{x}_{k}, m_{k}^{j} \mid \mathbf{z}_{1: k}\right) \\
& \propto \sum_{n=1}^{N} p\left(\mathbf{z}_{k} \mid \mathbf{x}_{k}^{j, n}, m_{k}^{j}\right) \bar{\mu}_{k}^{j, n} \delta\left(\mathbf{x}_{k}-\mathbf{x}_{k}^{j, n}\right) \Lambda_{k-1}^{j} .
\end{aligned}
$$

Thus, the samples remain the same, whereas the new weights can be updated as

$$
\mu_{k}^{j, n}=p\left(\mathbf{z}_{k} \mid \mathbf{x}_{k}^{j, n}, m_{k}^{j}\right) \Lambda_{k-1}^{j} \bar{\mu}_{k}^{j, n} /\left(\sum_{j=1}^{M} \sum_{n=1}^{N} \bar{\mu}_{k}^{j, n}\right) .
$$

\section{CASE STUDY}

A case study is designed to demonstrate the performance of the proposed algorithm. It is concerned with the arrival and landing of a UAV to a licensed uncontrolled aerodrome, e.g. Wellsbourne aerodrome in UK. In order to speed up its arrival the UAV is assumed to request a direct join on to the final. As the UAV is skipping the standard overhead join, it needs to be aware of other aircraft already in the circuit. To find an aircraft visually at distance using its zoom camera, an initial idea of the aircraft would be very helpful. This can be acquired when the traffic aircraft makes its downwind RT call exactly like the example in II, which gives a much better estimate of the aircraft's initial position.

This example scenario is illustrated in Fig 2, where three different circuit phases, namely downwind (Phase 3), turning into base (Phase 4) and the base leg (Phase 5), are considered during the simulation. It is assumed that at time $k=0$ a traffic aircraft is located in the downwind phase with the coordinates $(-1700 \mathrm{~m},-3300 \mathrm{~m})$ and the speed of $50 \mathrm{~m} / \mathrm{s}$. It follows the nominal circuit flight rules, i.e. with constant speed parallel to the runway in the downwind phase and then turns onto base with a turning rate about $3^{\circ} / \mathrm{s}$. Once established on the base leg indicated by the $90^{\circ}$ heading angle, it starts to decrease its speed at an acceleration of $-1 \mathrm{~m} / \mathrm{s}^{2}$.

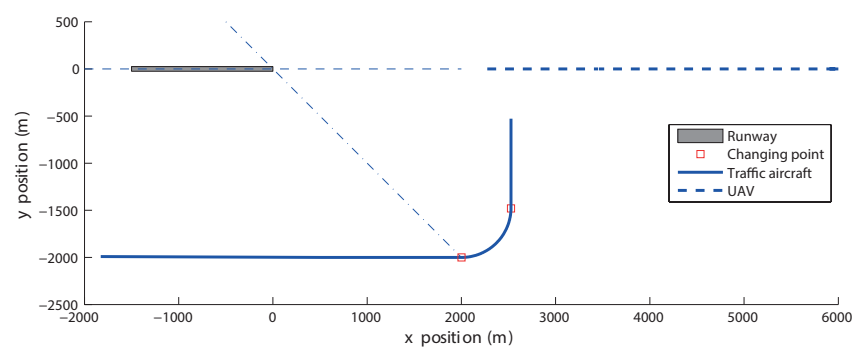

Fig. 2: Target tracking scenario

Following the general rules of air, the nominal transition criteria for turning into base can be described by the $45^{\circ}$ line which can be written as $\mathcal{C}_{34}=x_{k}+y_{k} \geq 0$. Since a human pilot may not be able to fly the circuit perfectly, a random variable $\gamma_{34}$ is introduced to characterise the stochastic transition behaviour. Thus, the state-dependent transition criteria can be modelled as $\mathcal{C}_{34}=\left\{x_{k}+y_{k} \geq \gamma\right\}$, where the random variable is assumed to follow a Gaussian distribution with a zero mean, i.e. $\gamma_{34} \sim \mathcal{N}\left(\gamma ; 0, \sigma_{34}^{2}\right)$. The standard deviation in the simulation is chosen to be $\sigma_{1}=200 \mathrm{~m}$ to capture the uncertainty in making the turning decision. The associated state-dependent transition probability can be evaluated as $\pi_{34}\left(\mathbf{x}_{k}\right)=\operatorname{Pr}\left\{\mathcal{C}_{34} \mid \mathbf{x}_{k}\right\}=\Phi\left(\mathcal{C}_{34} ; 0, \sigma_{34}^{2}\right)$. Similarly, the transition criteria from mode 4 to mode 5 can be written as $\mathcal{C}_{45}=\arctan \left(\frac{\dot{y}_{k}}{\dot{x}_{k}}\right)-85 \geq+\gamma_{45}$, where the Gaussian random variable $\gamma_{45}$ is zero mean with standard deviation of $5^{\circ}$. To capture any exception in modelling the stochastic transition modelling, a small number of transition probabilities are added into the theoretical ones. Therefore, the state-dependent transition probability matrix $\pi\left(\mathbf{x}_{k}\right) \in$ 
$\mathbb{R}^{3 \times 3}$ used in this case study can be defined as $\pi_{11}=[1-$ $\left.\Phi\left(\mathcal{C}_{34} ; 0, \sigma_{34}^{2}\right)\right] / \kappa, \pi_{12}=\left[\Phi\left(\mathcal{C}_{34} ; 0, \sigma_{34}^{2}\right)+0.005\right] / \kappa, \pi_{13}=$ $0.005 / \kappa, \pi_{21}=0.005 / \kappa, \pi_{22}=\left[1-\Phi\left(\mathcal{C}_{45} ; 0, \sigma_{34}^{2}\right)\right] / \kappa$, $\pi_{23}=\left[\Phi\left(\mathcal{C}_{45} ; 0, \sigma_{45}^{2}\right)+0.005\right] / \kappa$ and for the last row $\pi_{31}=$ $0.005, \pi_{32}=0.005$ and $\pi_{33}=0.99$. The normalisation factor can be calculated to be $\kappa=1.01$.

In the case study, the proposed SDT IMM particle filter is compared with traditional Markov jump based IMM particle filters with constant state transition matrices. Two filters (denoted as IMM-PF-1 and IMM-PF-2, respectively) are considered where the the mode transition matrices are

$$
\Pi_{1}=\left[\begin{array}{lll}
0.96 & 0.02 & 0.02 \\
0.02 & 0.96 & 0.02 \\
0.02 & 0.02 & 0.96
\end{array}\right] \Pi_{2}=\left[\begin{array}{lll}
0.8 & 0.1 & 0.1 \\
0.1 & 0.8 & 0.1 \\
0.1 & 0.1 & 0.8
\end{array}\right]
$$

where $\Pi_{2}$ represents a higher chance of transition than $\Pi_{1}$.

In designing filters, the process noises for different flight modes are set as zero mean Gaussian with different covariance matrices, i.e. $\sigma_{c v}=\operatorname{diag}\left\{(1 \mathrm{~m} / \mathrm{s})^{2},(0.5 \mathrm{~m} / \mathrm{s})^{2}\right\}$, $\sigma_{c t}=\operatorname{diag}\left\{(1.5 \mathrm{~m} / \mathrm{s})^{2},(1.5 \mathrm{~m} / \mathrm{s})^{2}\right\}$ and $\sigma_{w a}=$ $\operatorname{diag}\left\{(0.5 \mathrm{~m} / \mathrm{s})^{2},(1.5 \mathrm{~m} / \mathrm{s})^{2}\right\}$, respectively. It can be noted that for downwind (CV) and base (WA) modes the covariances are directional to reflect the corresponding directions of motion. The observation noise $\mathbf{v}_{k}$ is also assumed to be a Gaussian random variable which has zero mean and covariance matrix $\sigma_{v}=\operatorname{diag}\left\{(200 \mathrm{~m})^{2},(300 \mathrm{~m})^{2},\left(1^{\circ}\right)^{2}\right\}$. Moreover, to comply with the stall speed the minimum forward speeds in those models is assumed to be $20 \mathrm{~m} / \mathrm{s}$. More importantly the lateral velocities in those two modes are constrained to represent the aircraft flying approximately parallel to the runway on downwind and perpendicular to it on base by restricting the directions within $10^{\circ}$ variation. These state constraints can be incorporated in the particle filter by using the constrained likelihood function [17].

To represent the aircrafts unknown position prior to the downwind call the initial state distribution will be very uncertain. It is set to be Gaussian, with mean $\mathbf{x}_{0}=\left[\begin{array}{llll}-2500 & 50 & -3000 & 0\end{array}\right]^{T}$ and covariance $P_{0}=$ $\operatorname{diag}\{1000,10,1000,5\}$. The initial mode probabilities are $p\left(m_{0}^{1}\right)=1, p\left(m_{0}^{2}\right)=0$ and $p\left(m_{0}^{3}\right)=0$ which shows that after the RT call the filter is $100 \%$ certain that the aircraft is on the downwind leg. The sampling interval is $T=1$ second and the simulation runs for 140 time steps. The particle number used in the simulation is $N=4000$.

In the case study, Monte Carlo simulations were carried out for $L=100$ random generated tracks. Some example trajectories are given in Fig.3, where the mode changing points can be varied in a stochastic manner. The algorithm performances in terms of the tracking accuracy were evaluated by Root Mean Square Errors (RMSE) of the position and velocity. The time histories of RMSE are given in Fig. 4 and Fig. 5. The statistics of the MC simulation results are displayed in Table II, including average RMSE (aRMSE) for both position and velocity and the mode error count (MEC) of mode estimate.

It can be observed that the proposed SDT based IMMPF significantly outperforms the IMM-PFs based on constant

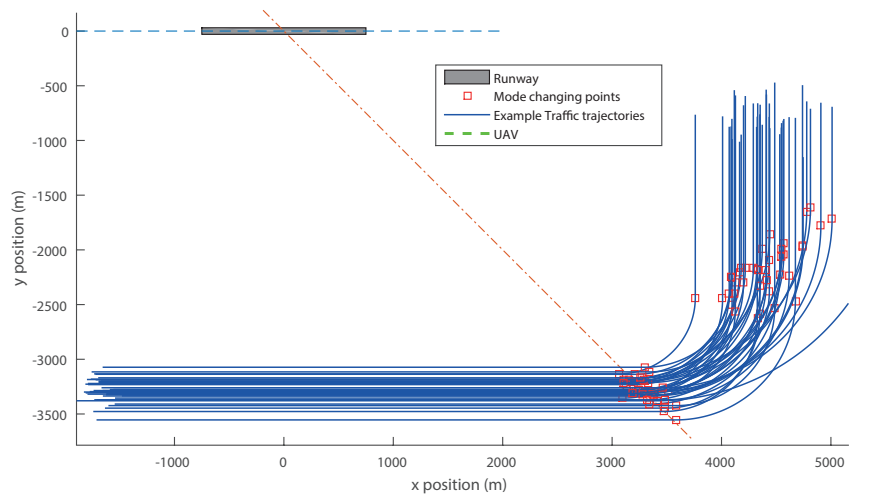

Fig. 3: Example trajectories for the MC simulation

transition probabilities because the circuit knowledge is more effectively incorporated in the filtering process in the proposed method. During the downwind phase the performance is similar but during mode 4 and 5 it is significantly better as the mode estimation is much more accurate for the SDTIMM.

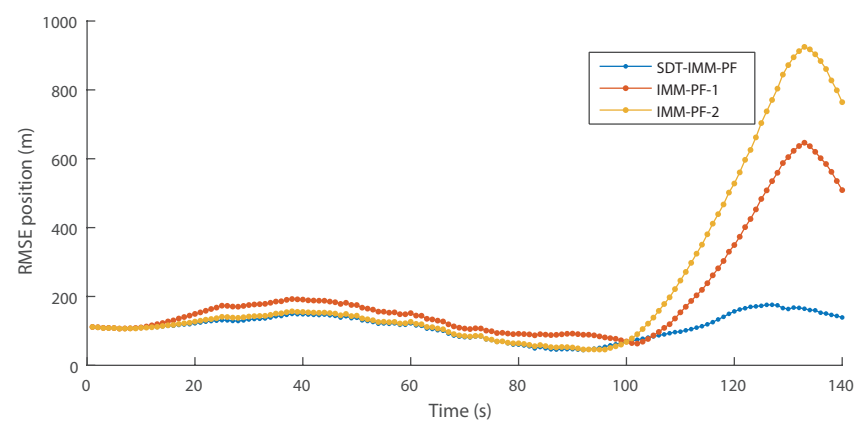

Fig. 4: RMSE of position estimation

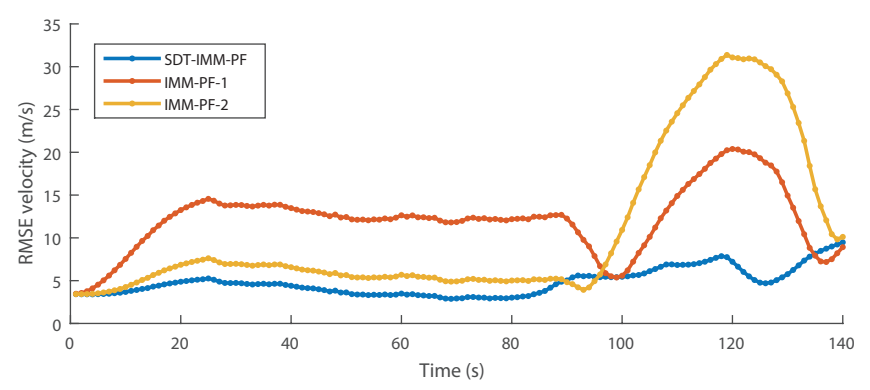

Fig. 5: RMSE of velocity estimation

The mode estimation accuracy is also an important measure of the performance. The estimated mode probabilities for a typical simulation run are shown in Fig. 6 It can be seen that the IMM-PF-1 with lower transition probability shows significant delays in estimating the mode transition, whereas the result from IMM-PF-2 is still susceptible to early mode change estimate, which is especially problematic for steadystate motion. The proposed SDT IMM particle filter on the other hand is able to demonstrate a better mode probability history. The counts of mode estimation errors during the simulation can be found in Table II. 
TABLE II: MC simulation results.

\begin{tabular}{l|c|c|c}
\hline Filters & aRMSE (pos.) & aRMSE (vel.) & MEC \\
\hline SDT-IMM-PF & $105 \mathrm{~m}$ & $4.8 \mathrm{~m} / \mathrm{s}$ & 7 \\
IMM-PF-1 & $192.6 \mathrm{~m}$ & $16.3 \mathrm{~m} / \mathrm{s}$ & 37.2 \\
IMM-PF-2 & $229 \mathrm{~m}$ & $10.5 \mathrm{~m} / \mathrm{s}$ & 47.4 \\
\hline
\end{tabular}

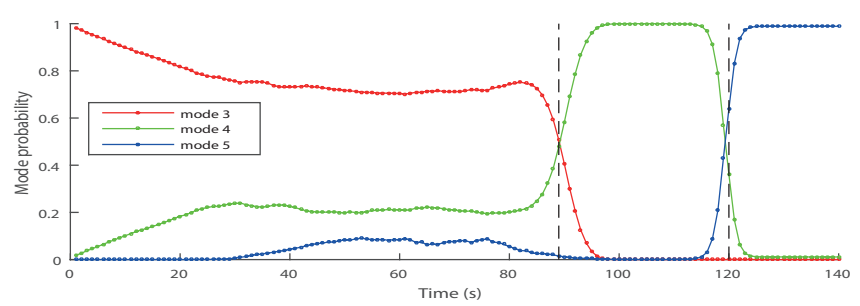

(a) Mode estimation of SDT-IMM-PF

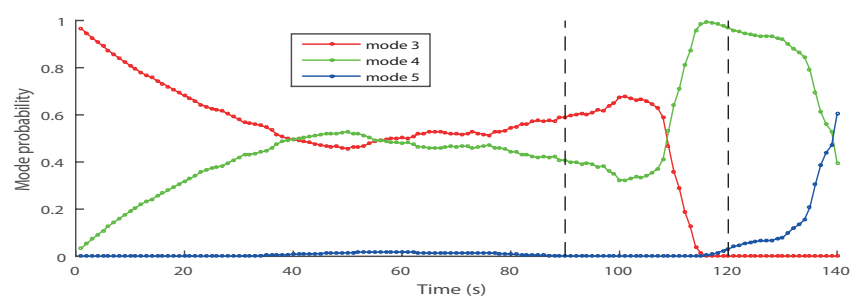

(b) Mode estimation of IMM-PF-1

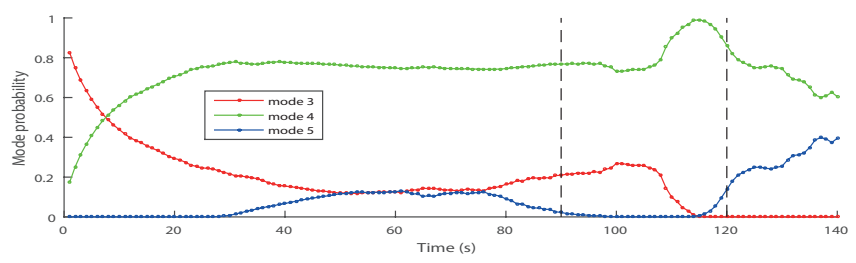

(c) Mode estimation of IMM-PF-2

Fig. 6: Mode estimation for a typical simulation run

\section{CONCLUSIONS}

Inserting a UAV into an uncontrolled aerodrome requires a good level of situation awareness on other traffic aircraft. In estimating the traffic aircraft's dynamical state and phase in the circuit, this paper investigates the effectiveness of incorporating the circuit flight rules into the traditional bearing-only target tracking. Multiple models are used to represent the different motion behaviours in different fight phases and the stochastic state-dependent transitions are used to characterise the actual phase transition with uncertainties. The resulted hybrid estimation problem is solved by the proposed SDT IMM particle filter, where the mode transition probabilities is calculated as a cumulative density function of system state instead of a constant matrix. This algorithm was compared with general Markov jump based IMM PFs in the case study. The MC simulations show that the proposed algorithm is not only able to obtain a better position and velocity accuracy but also a significantly improved mode estimate, which can more clearly indicate the intentions of the traffic aircraft. Future research following this work includes the further investigation on statistical modelling of the phase transition behaviours and incorporation of more realistic camera sensor model with data associated process.

\section{ACKNOWLEDGMENT}

This work was supported by the U.K. Engineering and Physical Sciences Research Council (EPSRC) Autonomous and Intelligent Systems programme under the grant number EP/J011525/1 with BAE Systems as the leading industrial partner.

\section{REFERENCES}

[1] CAA, "Cap 722 unmanned aircraft system operations in uk airspace," Tech. Rep., Civil Aviation Authority, 2012.

[2] Y. Bar-Shalom, X.R. Li, and T. Kirubarajan, Estimation with Applications to Tracking and Navigation: Theory Algorithms and Software, Wiley, 2004.

[3] Cunjia Liu, Matthew Coombes, Baibing Li, and Wen-Hua Chen, "Enhanced situation awareness for unmanned aerial vehicle operating in terminal areas with circuit flight rules," Proceedings of the Institution of Mechanical Engineers, Part G: Journal of Aerospace Engineering, to be published.

[4] M Sanjeev Arulampalam, Branko Ristic, N Gordon, and T Mansell, "Bearings-only tracking of manoeuvring targets using particle filters," EURASIP Journal on Applied Signal Processing, vol. 2004, pp. $2351-$ 2365, 2004.

[5] Branko Ristic, Sanjeev Arulampalam, and Neil Gordon, "Beyond the kalman filter," IEEE AEROSPACE AND ELECTRONIC SYSTEMS MAGAZINE, vol. 19, no. 7, pp. 37-38, 2004.

[6] E. Mazor, A. Averbuch, Y. Bar-Shalom, and J. Dayan, "Interacting multiple model methods in target tracking: a survey," Aerospace and Electronic Systems, IEEE Transactions on, vol. 34, no. 1, pp. 103-123, Jan 1998.

[7] Y. Bar-Shalom, S. Challa, and H.A.P. Blom, "Imm estimator versus optimal estimator for hybrid systems," Aerospace and Electronic Systems, IEEE Transactions on, vol. 41, no. 3, pp. 986-991, July 2005.

[8] M.S. Arulampalam, N. Gordon, M. Orton, and B. Ristic, "A variable structure multiple model particle filter for gmti tracking," in Information Fusion, 2002. Proceedings of the Fifth International Conference on, July 2002, vol. 2, pp. 927-934 vol.2.

[9] Y. Boers and J.N. Driessen, "Interacting multiple model particle filter," Radar, Sonar and Navigation, IEE Proceedings -, vol. 150, no. 5, pp. 344-349, Oct 2003.

[10] C.E. Seah and Inseok Hwang, "State estimation for stochastic linear hybrid systems with continuous-state-dependent transitions: An imm approach," Aerospace and Electronic Systems, IEEE Transactions on, vol. 45, no. 1, pp. 376-392, Jan 2009.

[11] C.E. Seah and Inseok Hwang, "Stochastic linear hybrid systems: Modeling, estimation, and application in air traffic control," Control Systems Technology, IEEE Transactions on, vol. 17, no. 3, pp. 563575, May 2009.

[12] H.A.P. Blom and E.A. Bloem, "Exact bayesian and particle filtering of stochastic hybrid systems," Aerospace and Electronic Systems, IEEE Transactions on, vol. 43, no. 1, pp. 55-70, January 2007.

[13] CAA, "Guide to visual flight rules in the uk," Tech. Rep., Civil Aviation Authority, 2011.

[14] FAA, "Advisory circular: Recommended standard traffic pattens and practices for aeronautical operations at airports with operating control towers," Tech. Rep., FAA, 1993.

[15] X.R. Li and V.P. Jilkov, "Survey of maneuvering target tracking. part i. dynamic models," Aerospace and Electronic Systems, IEEE Transactions on, vol. 39, no. 4, pp. 1333-1364, Oct 2003.

[16] Yan Zhang and Yaakov Bar-Shalom, "Tracking move-stop-move targets with state-dependent mode transition probabilities," Aerospace and Electronic Systems, IEEE Transactions on, vol. 47, no. 3, pp. 2037-2054, July 2011.

[17] Xinguang Shao, Biao Huang, and Jong Min Lee, "Constrained bayesian state estimation a comparative study and a new particle filter based approach," Journal of Process Control, vol. 20, no. 2, pp. 143 - 157, 2010. 